\title{
Editorial
}
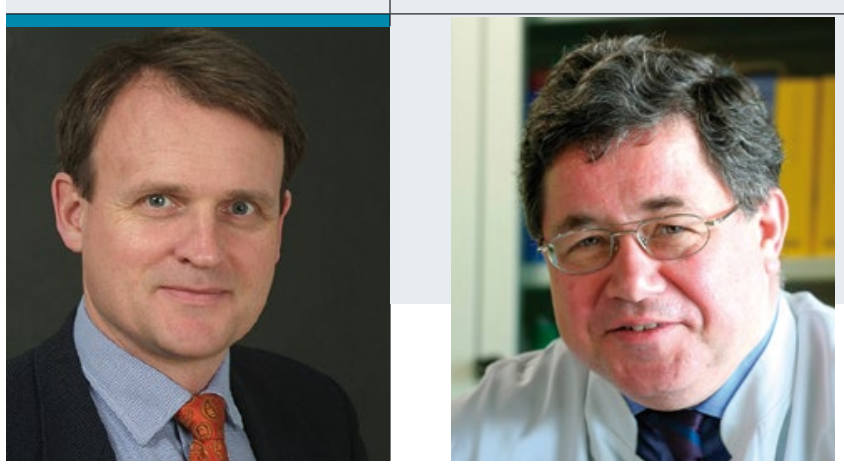

„Positive Signale konnten wir kürz-

lich aus dem Bundesministerium

für Gesundheit vernehmen."

Prof. Dr. Thilo Jakob, Klinik für Dermatologie und Allergologie, Universitätsklinikum Gießen und Marburg, Standort Gießen

Prof. Dr. Hans F. Merk, Hautklinik der Medizinischen Fakultät, Universitätsklinikum der RWTH Aachen

\section{Verbandsarbeit trägt Früchte}

$\mathrm{P}$ assend zur Jahreszeit bieten wir Ihnen einen bunten Blumenstrauß allergologischer Themen: Als Ursache für die deutliche Zunahme allergischer Erkrankungen in den letzten Jahrzehnten werden Veränderungen im Lebensstil angeschuldigt. Viele dieser Veränderungen können sich direkt oder indirekt auf die Zusammensetzung des intestinalen Mikrobioms auswirken. Ein heiß diskutiertes Thema ist daher die Frage, ob Mikroorganismen im Darm die Entstehung, Auslösung und Chronifizierung allergischer Erkrankungen beeinflussen. Welche Daten diese Hypothese unterstützen und welche Mechanismen beteiligt sein könnten, erfahren Sie in der Übersichtsarbeit von Dr. Casper Ohnmacht (S. 16). Für diejenigen, die sich nicht mit dem englischen Beitrag befassen wollen, haben wir die wesentlichen Punkte in einer deutschen Kurzfassung zusammengestellt (S. 12). Diese deutschen Referate haben wir auf Anregung einer interessierten Leserin eingeführt und sind damit auf sehr positive Resonanz gestoßen. Sollten Sie also selbst Vorschläge für das Allergo Journal haben, zögern Sie nicht, uns zu kontaktieren.

Im Zeitalter der evidenzbasierten Medizin muss man sich immer häufiger fragen, welche Bedeutung die Finanzierung der Evidenzgenerierung spielt. Die Frage „Who pays for the evidence?" scheint besonders dann relevant, wenn es um neue Krankheitsentitäten geht, deren Existenz in der Fachwelt unverändert kontrovers diskutiert werden. In diesem Zusammenhang ist der Im-Fokus-Beitrag von Dr. Imke Reese interessant, der eine viel diskutierte italienische Arbeit zur Nicht-Zöliakie-Gluten-Sensitivität kritisch durchleuchtet (S. 14). Im Gegensatz zum Studienfazit, kommt Dr. Reese zu dem Schluss, dass die Untersuchung keinen Beweis für die Existenz der Nicht-Zöliakie-Gluten-Sensitivität darstellt. "Ein Schelm, der Böses dabei denkt", wenn ausgerechnet die Hersteller von glutenfreien Backwaren Konsensuskonferenzen zu diesem Thema finanzieren.

Auch der nächste Beitrag bleibt beim Thema Backgewerbe: Bäckerasthma und Bäckerrhinitis können eine diagnostische Herausforderung darstellen. In der Orginalarbeit von Simonis et al. wird der Frage nachgegangen, welchen Stellenwert der BasophilenAktivierungstest hierbei spielen kann (S. 26). Zu guter Letzt können Sie sich im aktuellen CME-Beitrag von Prof. Dr. Karl-Christian Bergmann, Berlin, über den Einfluss von Wetterbedingungen und Klimawandel auf allergische Erkrankungen informieren.

Positive Signale konnten wir kürzlich aus dem Bundesministerium für Gesundheit vernehmen unsere langjährige Verbandsarbeit scheint erste Früchte zu tragen: Eine aktuelle Ausschreibung wird gezielt „Forschung zur Steigerung der Patientenkompetenz und Patientenorientierung in der Allergologie" fördern. Hier sollen über drei Jahre Projekte unterstützt werden, die das Bewusstsein für allergische Erkrankungen und die Krankenkompetenz stärken sowie die Beratungskompetenz des pharmazeutischen Personals in Apotheken und die der Ärzte optimieren. Ein weiteres positives Signal ist eine Initiative, in der ein mit Bundesmitteln gefördertes Internetportal zu Allergieerkrankungen erstellt werden soll. Vielleicht erinnern Sie sich noch an die Website des Bundesamts für Verbraucherschutz und Lebensmittelsicherheit zum Aktionsplan gegen Allergien (www.aktionsplan-allergien.de), das leider 2012 eingestellt wurde. In einer gemeinsamen Initiative des Robert Koch-Instituts, des Bundesamts für Risikobewertung und des Paul-Ehrlich-Instituts in Zusammenarbeit mit den allergologischen Fachgesellschaften sollen hier nun die Fäden wieder aufgenommen und eine zentrale und neutrale Informationsquelle zum Thema Allergien geschaffen werden. Als Fachgesellschaften begrüßen wir diese Art der Förderung ausdrücklich und sind gespannt auf die Ergebnisse.

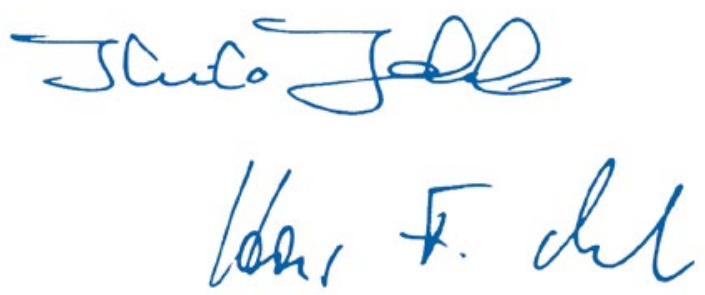

